\title{
Equity across the cancer care continuum for culturally and linguistically diverse migrants living in Australia: a scoping review
}

\author{
Brighid Scanlon ${ }^{1,2^{*}}$, Mark Brough $^{1}$, David Wyld ${ }^{1,2,3}$ and Jo Durham ${ }^{1}$
}

\begin{abstract}
International evidence suggests migrants experience inequitable access, outcomes and treatment quality across the cancer care continuum. There is currently limited research assessing equity across the cancer care continuum for culturally and linguistically diverse migrants living in Australia. A detailed protocol and search strategy were developed and used to identify all relevant literature, utilising the Joanna Briggs Institute Reviewer's Manual. Systematic searching was conducted via multiple databases and identified studies were screened against pre-identified inclusion and exclusion criteria. 71 studies met the inclusion criteria for analysis. Most studies examined cancer detection via screening. Very few studies examined cancer prevention, diagnosis, treatment or palliative care. Most studies focused on patient-sided barriers to care and there was a paucity of information regarding institutional barriers to health. Cancer-related outcomes were seldom examined, and most studies were qualitative or behavioral analysis. Results highlighted significant communication issues spanning the cancer care continuum and a context of inadequate support for both patients and clinicians. There is a demonstrable need to examine equity in access and outcomes for culturally and linguistically diverse cancer populations. This requires the identification of cancer-related disparities and an examination of institutional barriers to care. Through addressing this dearth of information, future research and health policy can support the operationalisation of health equity.
\end{abstract}

Keywords: Cancer, Disparities, Racialisation, Health Equity, Migrants, Culturally Diverse

\section{Background}

Global migration continues to play an important role in human history and is often influenced by periods of instability. Instability arises from factors such as weak governance, unequal distribution of resources, violent conflict, social injustice, persistent negative impact from disasters, including pandemics such as COVID-19,

\footnotetext{
* Correspondence: brighid.scanlon@hdr.qut.edu.au

'Queensland University of Technology, 149 Victoria Park Road, QLD 4059 Kelvin Grove, Australia

${ }^{2}$ Royal Brisbane and Women's Hospital, Butterfield Street, QLD 4029 Herston, Australia

Full list of author information is available at the end of the article
}

economic hardship and poverty [1, 2]. Currently, over 272 million migrants have sought safety or opportunity in new countries and states, influencing the cultural and linguistic diversity of many regions [3]. As the COVID19 pandemic demonstrates, disruptive events can expose existing inequities, or create new inequities, in areas such as health. Migrants from culturally and linguistically diverse (CALD) backgrounds may be particularly impacted, as the health systems they find themselves reliant on are often based on a culturally dominant model of care [4]. This approach is not responsive to the needs of diverse or minority populations and thus can produce and reproduce health inequities. It is therefore 
imperative that multilingual and multi-ethnic societies, such as Australia, strive for the promotion of health equity for all individuals [5]. Whilst the term 'CALD' is common within the Australian literature, the most consistent term used in the international literature is 'ethnic minority'. Due to this, both terms were examined within this review. This research was conducted with consideration of the Australian historical backdrop of invasion, colonisation and dispossession of Aboriginal and Torres Strait Islander peoples. Thus, terminology such as 'migrant', 'ethnic minority' and 'CALD' have been utilised with caution and respect.

Health disparities exist both within and between countries [6] and in a globalised world, it is imperative to consider the health effects of differing disease burdens on migrant populations. Within countries of the Organization for Economic Co-operation and Development (OECD) and emerging economies, demographic and epidemiological transitions are underway, characterised by an aging population and a high burden of non-communicable diseases; including cancer [7-9]. A systematic analysis of 195 countries displayed that between the years 2006 and 2016, incident cases for all cancers increased significantly [7]. However, the burden of certain cancer types differs greatly between countries, for example cancers with infectious etiologies such as cervical, liver and gastric cancers are overrepresented in those from lower-middle income countries [7, 10]. In addition, effective cancer detection and treatment requires significant resource allocations and sophisticated diagnostic and therapeutic services and thus vary greatly between countries [11]. These factors influence health disparities between countries and must be considered when providing equitable and responsive cancer care in the post-migratory context [12].

Within high-income countries inequities across the cancer care continuum are a pertinent exemplar of the widening of health disparities for CALD populations [13]. The chronicity and complexity of the cancer journey makes it a valuable measure for health disparity research, with disparities in access and outcomes being mirrored in other chronic diseases affecting ethnic minority populations, such as cardiovascular disease and diabetes mellitus [14]. Research from the United States has displayed significant disparities across the cancer care continuum for ethnic minority populations, including in African American, Asian American, Latino/Hispanic and Pacific Islander populations [15]. In the United States, cancer is the leading cause of death for Latino people, with Latino women experiencing the highest cervical cancer rates, with incidence $64 \%$ higher than 'non-Hispanic white women' [15]. American-Samoan men are eight-times more likely to develop liver cancer and American-Samoan women are twice as likely to develop, and die, from cervical cancer than 'non-Hispanic white women' [15]. Canadian research also demonstrates disparities for ethnic minority populations in access to screening, follow-up of abnormal findings, length of survival, quality of life, adherence to treatment regimens and quality of interactions with physicians $[16,17]$.

Health disparities have been defined as,...a particular type of health difference closely linked with economic, social, or environmental disadvantage. Health disparities adversely affect groups of people who have systematically experienced greater social or economic obstacles to health...[18].

Disparities in cancer care that disproportionately affect racialised groups are no longer considered the result of biological or behavioural mechanisms, but of socially and politically constructed identities that reflect power differentials and discrimination throughout society [19]. The powerful effects of these processes create macro societal and micro institutional barriers, which can manifest as institutional racism, fragmented health systems that are challenging to navigate, a lack of appropriate and accessible health information, high out-of-pocket expenditure and an environment of poor communication [13, 20, 21]. Despite this, health disparity research continues to perpetuate an individualistic, deficit perspective that fails to acknowledge the structural and institutional drivers of inequity.

Historically, the reduction of health disparities has been the predominant focus of research, however, recently attention has turned to promoting equity $[4,22]$. Health equity has been defined as "...the principle underlying a commitment to reduce, and ultimately eliminate disparities in health and in its determinants" [23]. A useful way to examine equity throughout the multiple stages of cancer is to utilise the cancer care continuum framework. Developed in the $1970 \mathrm{~s}$, this framework describes the various stages of cancer care, including prevention, detection, diagnosis, treatment and survivorship [24]. An important omission of the framework is its lack of attention to end-of-life or palliative care, which will be included in this review as an additional stage of the cancer care continuum. To date, whilst there has been some research into cancer-related health disparities for CALD populations in Australia, there is a distinct lack of attention to the diverse and changing needs of populations across the cancer care continuum. This is significant, as Australia is a multiethnic and multilingual society and therefore health services must adapt to the changing needs of populations.

The intent of this review is to synthesise what is currently known about equity across the cancer care continuum for CALD migrant populations in Australia and to identify areas in need of further research. This national focus acknowledges that whilst the needs of 
specific populations may differ, there is a need to develop health services that are adaptive and responsive to the changing needs of populations.

\section{Main Text}

\section{Literature review process}

A scoping review was deemed the most appropriate method, due to the scarcity of high-quality published information on the topic. This allowed for a comprehensive search of all available information and the identification of research gaps. The scoping review followed the guidelines provided in the Joanna Briggs Institute (JBI) Reviewer's Manual and complied with the PRISMA extension for scoping reviews, PRISMA-ScR Guidelines [25].

To be included in this review papers needed to (a) report one or multiple phases of the cancer continuum, such as prevention, detection, diagnosis, treatment, survivorship or the additional phase proposed by the lead researcher; palliative care, or (b) discuss a common phenomenon present in multiple phases, or (c) discuss the perspectives of CALD migrants, clinicians, or migrants' families and carers; (d) publish between 2000 and 2020 for currency; and (e) be written in English. The term 'CALD migrants' was used to define people who were born in countries where English is not a main language and who are also considered an ethnic or cultural minority in Australia. Whilst acknowledging the problematic nature of this terminology, it is currently in line with the Australian research landscape [26]. Qualitative, quantitative and mixed-method studies were included for comprehensiveness. No studies were excluded based upon quality. Studies were excluded if they did not report on CALD migrant populations, or their families, or the healthcare professionals interacting directing with them. Studies were excluded if they included nonmalignant diseases or conditions. Studies were excluded if they grouped CALD populations with Australian-born participants, such as Aboriginal and Torres Strait Islander peoples. Studies published earlier than the year 2000 were excluded.

A comprehensive search strategy was developed in accordance with the JBI reviewer's manual and the PRIS MA ScR guidelines [25]. The search was designed to capture all relevant studies that combined the concepts of 'CALD migrants', 'cancer care', 'access' and 'equity' in the Australian healthcare system. The following databases were searched between 2000-2020:

- PubMed Central.

- CINAHL EBSCO.

- PsycInfo Ovid.

- Cochrane Library.

- Joanna Briggs Institute EDP Database.
- ProQuest Dissertation and Theses (Grey literature).

After implementing the search protocol, the final search results were exported to Endnote and duplicates removed. The abstracts and full text articles identified through the search strategy were screened for relevance by the lead researcher and then by a second researcher, based on the inclusion and exclusion criteria. The search strategy is displayed in Fig. 1. Citations were managed using Endnote X9 and a Microsoft document. Discrepancies between authors were resolved through discussion. Included studies were charted using a data extraction form based on JBI Reviewer's Manual, see in Table 1 [25].

Data were extracted into an Excel spreadsheet and descriptively mapped to demonstrate the current landscape of the literature, rather than assessed analytically [25]. Frequency counts of key concepts, populations and characteristics of the current studies were completed. Data were disaggregated into the stages of the cancer care continuum to show the distribution of the studies across the continuum. The extracted data, shown in Table 2, were used to formulate tables and charts mapping the studies' distribution based on geographic location, CALD group studied, sample size, year published, methodologies and main findings and to identify research gaps.

\section{Findings}

The search strategy identified 188 published studies. After removing duplicates there were 123 studies. After screening results by title and abstract there were 77 studies. After reading full-text articles, 71 studies met the inclusion criteria and were included in the final analysis. Details of the data extraction process are shown in Table 1. Figure 2. displays the distribution of the studies based on the stages of the cancer care continuum.

\section{Disparities in screening for prevention and detection}

Two studies recommended targeted screening for migrants from hepatitis B and Helicobacter pylori endemic countries as a mode of cancer prevention [27, 28]. Those with chronic hepatitis $\mathrm{B}(\mathrm{CHB})$ are 6-12 times more likely to develop liver cancer and those from Helicobacter pylori $(H$. pylori.) endemic countries are at significantly higher risk of developing gastric cancers $[27,28]$. Thus, through addressing disparities in $\mathrm{CHB}$ and $H$. pylori, cancer prevention can also be achieved for some migrant groups.

The detection phase of the cancer care continuum was measured through screening services and reported in 


\section{STUDY DISTRIBUTION ACROSS THE CANCER CARE CONTINUUM}

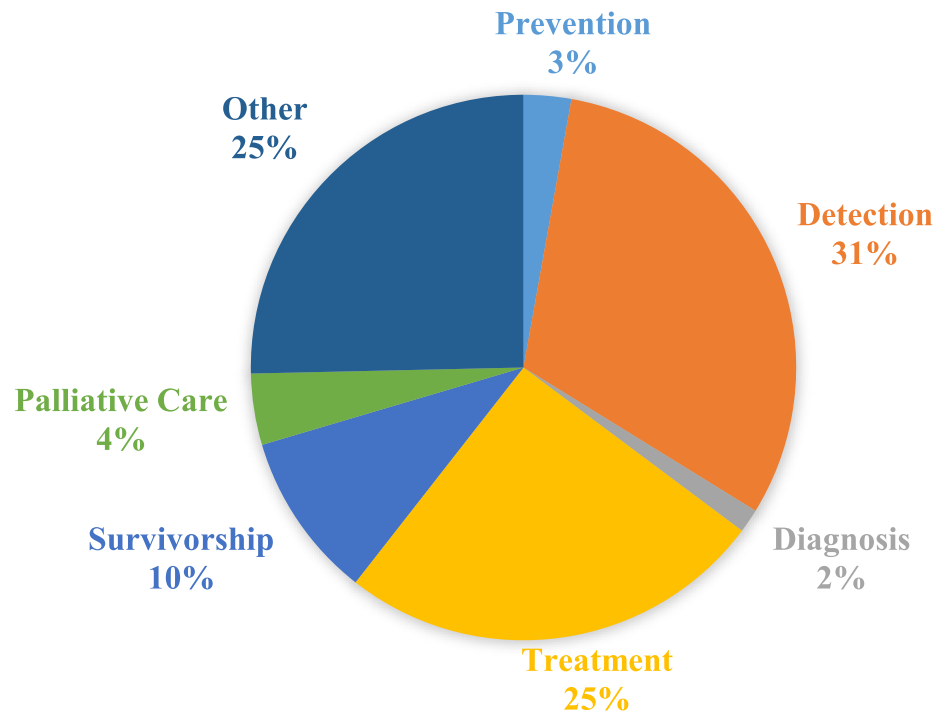

Fig. 1 Study distribution across the cancer care continuum

twenty-two studies [29-50]. Six studies reported on the barriers and/or facilitators to screening services for cervical, breast and colorectal cancer [30-32, 39-41]. Common barriers identified were lack of screening knowledge, feelings of embarrassment, fear over the gender of health provider, privacy concerns, religious and cultural beliefs, language barriers, fatalistic views of cancer, screening not being promoted in community languages and location of services $[30-32,39,40]$. These findings largely perpetuated the individualistic, deficit perspective of barriers to care, with institutional barriers less often reported. Strong facilitators for screening were a doctor's recommendation, having a female doctor of the same ethnic background, feeling understood by health providers and having strong social support [32, 39].

Ten studies focused on screening participation rates for breast, bowel and cervical cancers [33, 34, 36-38, 42, 44, 47-49]. A study of more than 24,000 women found that migrant women born in Middle Eastern and Asian regions were less likely to participate in cervical cancer screening than Australian-born women [37]. Similarly,

Table. 1 Data extracted from studies

\begin{tabular}{ll}
\hline Data extracted & \\
\hline Author & Location \\
Year & Sample Size \\
Cancer Care Continuum Stage Assessed & Population Group \\
Study Design & Methodology \\
Aims/Purpose & Key Findings \\
\hline
\end{tabular}

women from African refugee backgrounds were significantly less likely to utilise cervical cancer screening services [49]. Factors associated with higher screening participation were length of residency in Australia, marital and employment status, flexibility of clinic hours, gender of the health practitioner, having a reminder system and accessible health information $[42,48]$.

\section{Diagnosis and treatment participation}

Only one study examined the diagnostic pathway for CALD populations [51]. The LEAD protocol details a prospective observational cohort study to compare the diagnostic pathways for lung cancer between CALD and 'Anglo-Australian' populations, with no results available to date [51]. Eighteen studies analysed the treatment stage of the cancer care continuum [5, 12, 52-67]. Of these, four examined treatment coordination [52, 60, 63, 67]. A randomised control trial found calling women for appointment reminders in their preferred language significantly increased appointment and attendance rates and was more successful than translated reminder letters [52]. Shaw et al. (2016) described the experiences of cancer care coordination for Chinese, Arabic and Macedonian speaking patients, finding these migrants require additional assistance navigating the health system and information appropriate to their linguistic background [63].

Only three studies reported on equity in treatment outcomes $[5,12,62]$. Two of these compared the outcomes of Arabic, Chinese and Greek populations to that of Anglo-Australians and found the former had significantly worse health-related quality of life and higher 
Table. 2 Sample of data extraction and charting process

\begin{tabular}{|c|c|c|c|c|c|c|c|c|c|}
\hline Author & Year & CCC Stage & $\begin{array}{l}\text { Study } \\
\text { Design }\end{array}$ & Aims/Purpose & Location & Sample Size & $\begin{array}{l}\text { Population } \\
\text { Group }\end{array}$ & Methodology & Key Findings \\
\hline $\begin{array}{l}\text { Robotin, } \\
\text { M C. et al. }\end{array}$ & 2010 & Prevention & $\begin{array}{l}\text { Cohort } \\
\text { study }\end{array}$ & $\begin{array}{l}\text { Aiming to } \\
\text { optimise the } \\
\text { management of } \\
\text { chronic } \\
\text { Hepatitis B in } \\
\text { at-risk popula- } \\
\text { tions via screen- } \\
\text { ing, surveillance } \\
\text { and treatment. } \\
\text { To prevent de- } \\
\text { velopment of } \\
\text { liver disease } \\
\text { and liver cancer. }\end{array}$ & $\begin{array}{l}\text { South-West } \\
\text { Sydney, New } \\
\text { South Wales, } \\
\text { Australia }\end{array}$ & $\begin{array}{l}5,800 \text { local } \\
\text { residents } \\
\text { (hypothetical } \\
\text { cohort) }\end{array}$ & $\begin{array}{l}\text { Migrants from } \\
\text { Hepatitis B } \\
\text { endemic } \\
\text { countries }\end{array}$ & $\begin{array}{l}\text { Estimated } \\
\text { numbers of CHB } \\
\text { infections were } \\
\text { derived from } \\
\text { Australian } \\
\text { Bureau of } \\
\text { Statistics data. } \\
\text { These figures } \\
\text { were entered } \\
\text { into a Markov } \\
\text { model of } \\
\text { disease } \\
\text { progression, } \\
\text { constructing a } \\
\text { hypothetical } \\
\text { cohort. The } \\
\text { stages of CHB } \\
\text { were calculated, } \\
\text { as well as the } \\
\text { primary and } \\
\text { specialist } \\
\text { healthcare } \\
\text { resources } \\
\text { needed } \\
\text { annually by the } \\
\text { cohort }\end{array}$ & $\begin{array}{l}\text { 1. There is a } \\
\text { limited increase } \\
\text { in GP } \\
\text { consultations, a } \\
\text { moderate } \\
\text { increase in } \\
\text { specialist } \\
\text { consultations and } \\
\text { a large increase } \\
\text { in liver } \\
\text { ultrasounds for } \\
\text { this cohort } \\
\text { annually2. New } \\
\text { models of care } \\
\text { are required in } \\
\text { order to scale up } \\
\text { the existing } \\
\text { services available } \\
\text { for CHB } \\
\text { population }\end{array}$ \\
\hline $\begin{array}{l}\text { Schulz, T } \\
\text { R. et al. }\end{array}$ & 2014 & Prevention & $\begin{array}{l}\text { Cost- } \\
\text { Benefit } \\
\text { Analysis }\end{array}$ & $\begin{array}{l}\text { To compare if } \\
\text { screening for } \\
\text { and eradication } \\
\text { of Helicobacter } \\
\text { pylori in } \\
\text { immigrants } \\
\text { reduces burden } \\
\text { of gastric } \\
\text { cancer }\end{array}$ & $\begin{array}{l}\text { Melbourne, } \\
\text { Victoria, } \\
\text { Australia }\end{array}$ & N/A & $\begin{array}{l}\text { Asymptomatic } \\
\text { immigrants } \\
\text { from high } \mathrm{H} \text {. } \\
\text { pylori } \\
\text { prevalence } \\
\text { areas }\end{array}$ & $\begin{array}{l}\text { Nine different } \\
\text { screening and } \\
\text { follow-up strat- } \\
\text { egies were } \\
\text { compared with } \\
\text { the current } \\
\text { process of no } \\
\text { screening }\end{array}$ & $\begin{array}{l}\text { 1. H. pylori } \\
\text { screening and } \\
\text { eradication can } \\
\text { be an effective } \\
\text { strategy for } \\
\text { reducing rates of } \\
\text { gastric cancer2. } \\
\text { Data suggest that } \\
\text { use of stool } \\
\text { antigen testing is } \\
\text { the most cost- } \\
\text { effective } \\
\text { approach }\end{array}$ \\
\hline $\begin{array}{l}\text { Aminisani, } \\
\text { N. et al. }\end{array}$ & -2012 & $\begin{array}{l}\text { Detection } \\
\text { (Screening) }\end{array}$ & $\begin{array}{l}\text { Cohort } \\
\text { Study }\end{array}$ & $\begin{array}{l}\text { To assess the } \\
\text { Cervical Cancer } \\
\text { Screening } \\
\text { behaviours of } \\
\text { migrants, } \\
\text { compared to } \\
\text { Australian-born } \\
\text { women }\end{array}$ & $\begin{array}{l}\text { New South } \\
\text { Wales, } \\
\text { Australia }\end{array}$ & $\begin{array}{l}\text { 12, } 541 \\
\text { migrants } \\
\text { linked to } 12, \\
143 \\
\text { Australian- } \\
\text { born women }\end{array}$ & $\begin{array}{l}\text { Middle Eastern } \\
\text { or Asian-born } \\
\text { women aged } \\
\text { between } 20- \\
54 \text { years }\end{array}$ & $\begin{array}{l}\text { Year } 2000 \\
\text { records of NSW } \\
\text { Midwives Data } \\
\text { Collection } \\
\text { (country of } \\
\text { birth) linked } \\
\text { with Cervical } \\
\text { Screening } \\
\text { Register }\end{array}$ & $\begin{array}{l}\text { 1. Migrant } \\
\text { women are less } \\
\text { likely to } \\
\text { participate in } \\
\text { Cervical } \\
\text { Screening than } \\
\text { Australian-born } \\
\text { women at the } \\
\text { recommended } \\
\text { interval2. Migrant } \\
\text { women's screen- } \\
\text { ing is less related } \\
\text { to socio- } \\
\text { economic status, } \\
\text { smoking and par- } \\
\text { ity as Australian- } \\
\text { born women }\end{array}$ \\
\hline $\begin{array}{l}\text { Aminisani, } \\
\text { N. et al. }\end{array}$ & 2012 & $\begin{array}{l}\text { Detection } \\
\text { (Screening) }\end{array}$ & $\begin{array}{l}\text { Cohort } \\
\text { Study }\end{array}$ & $\begin{array}{l}\text { To assess the } \\
\text { Cervical } \\
\text { Screening } \\
\text { behaviours of } \\
\text { older migrant } \\
\text { women, } \\
\text { compared to } \\
\text { Australian-born } \\
\text { women }\end{array}$ & $\begin{array}{l}\text { New South } \\
\text { Wales, } \\
\text { Australia }\end{array}$ & $\begin{array}{l}14,228 \\
\text { migrants } \\
\text { linked to } 13, \\
939 \\
\text { Australian- } \\
\text { born women }\end{array}$ & $\begin{array}{l}\text { Middle Eastern } \\
\text { or Asian-born } \\
\text { women aged } \\
\text { between } 40- \\
64 \text { years }\end{array}$ & $\begin{array}{l}\text { Year 2000-2001 } \\
\text { records were } \\
\text { compared to an } \\
\text { age and area } \\
\text { matched } \\
\text { random sample } \\
\text { of Australian- } \\
\text { born women } \\
\text { through the } \\
\text { NSW Admitted }\end{array}$ & $\begin{array}{l}\text { 1. Older women } \\
\text { from the Middle } \\
\text { East, North East } \\
\text { and South East } \\
\text { Asia appeared to } \\
\text { have similar } \\
\text { overall screening } \\
\text { participation to } \\
\text { that of Australian- } \\
\text { born women2. }\end{array}$ \\
\hline
\end{tabular}


Table. 2 Sample of data extraction and charting process (Continued)

\begin{tabular}{|c|c|c|c|c|c|c|c|c|c|}
\hline Author & Year & CCC Stage & $\begin{array}{l}\text { Study } \\
\text { Design }\end{array}$ & Aims/Purpose & Location & Sample Size & $\begin{array}{l}\text { Population } \\
\text { Group }\end{array}$ & Methodology & Key Findings \\
\hline & & & & & & & & $\begin{array}{l}\text { Patients Data } \\
\text { Collection and } \\
\text { Cervical Screen- } \\
\text { ing Registers }\end{array}$ & $\begin{array}{l}\text { Women from } \\
\text { South Central } \\
\text { Asia appeared } \\
\text { less likely than } \\
\text { Australian-born } \\
\text { women to partici- } \\
\text { pate in cervical } \\
\text { screening at the } \\
\text { recommended } \\
\text { interval }\end{array}$ \\
\hline Author & Year & CCC Stage & $\begin{array}{l}\text { Study } \\
\text { Design }\end{array}$ & Aims/Purpose & Location & Sample Size & $\begin{array}{l}\text { Population } \\
\text { Group }\end{array}$ & Methodology & Key Findings \\
\hline $\begin{array}{l}\text { Aminisani, } \\
\text { N. et al. }\end{array}$ & 2012 & $\begin{array}{l}\text { Detection } \\
\text { (Screening) }\end{array}$ & $\begin{array}{l}\text { Cohort } \\
\text { Study }\end{array}$ & $\begin{array}{l}\text { To compare the } \\
\text { rates of cervical } \\
\text { cancer in } \\
\text { migrant and } \\
\text { Australian-born } \\
\text { women after } \\
\text { the introduction } \\
\text { of Organised } \\
\text { Cervical } \\
\text { Screening }\end{array}$ & $\begin{array}{l}\text { New South } \\
\text { Wales, } \\
\text { Australia }\end{array}$ & $\begin{array}{l}11,485 \\
\text { women }\end{array}$ & $\begin{array}{l}\text { Women aged } \\
15+ \\
\text { diagnosed } \\
\text { with invasive } \\
\text { cervical cancer } \\
\text { between } \\
\text { 1973-2008 }\end{array}$ & $\begin{array}{l}\text { Joinpoint } \\
\text { regression was } \\
\text { used to assess } \\
\text { the annual } \\
\text { percentage } \\
\text { changes in } \\
\text { incidence and } \\
\text { mortality before } \\
\text { and after the } \\
\text { introduction of } \\
\text { Organised } \\
\text { Cervical } \\
\text { Screening in } \\
1991\end{array}$ & $\begin{array}{l}\text { 1. Incidence and } \\
\text { mortality rates fell } \\
\text { post the } \\
\text { introduction of } \\
\text { Organised } \\
\text { Cervical } \\
\text { Screening for } \\
\text { Australian-born, } \\
\text { UK-born, Ireland- } \\
\text { born women, } \\
\text { and to a lesser } \\
\text { extent woman } \\
\text { from the Middle } \\
\text { East, New Zea- } \\
\text { land, North Africa } \\
\text { and Asian-born } \\
\text { women.2. There } \\
\text { was a rise in mor- } \\
\text { tality found in } \\
\text { women from a } \\
\text { "rest of world" } \\
\text { category, that } \\
\text { may be explained } \\
\text { by recent } \\
\text { migration }\end{array}$ \\
\hline $\begin{array}{l}\text { Anaman, J } \\
\text { A.et al. }\end{array}$ & 2017 & $\begin{array}{l}\text { Detection } \\
\text { (Screening) }\end{array}$ & $\begin{array}{l}\text { Cross- } \\
\text { sectional } \\
\text { Survey }\end{array}$ & $\begin{array}{l}\text { To compare the } \\
\text { level of cervical } \\
\text { screening } \\
\text { uptake between } \\
\text { refugee and } \\
\text { non-refugee Af- } \\
\text { rican immigrant } \\
\text { women }\end{array}$ & $\begin{array}{l}\text { Brisbane, } \\
\text { Queensland, } \\
\text { Australia }\end{array}$ & $\begin{array}{l}144 \text { African } \\
\text { Refugees, } \\
110 \text { African } \\
\text { non-refugees }\end{array}$ & $\begin{array}{l}254 \text { African } \\
\text { women aged } \\
\text { between } 21- \\
62 \text {, from } 22 \\
\text { African } \\
\text { countries }\end{array}$ & $\begin{array}{l}\text { Chi-Square tests } \\
\text { were used to } \\
\text { compare } \\
\text { demographic } \\
\text { and health- } \\
\text { related charac- } \\
\text { teristics be- } \\
\text { tween refugee } \\
\text { and non- } \\
\text { refugee women. } \\
\text { Multiple logistic- } \\
\text { regression ana- } \\
\text { lyses were per- } \\
\text { formed to } \\
\text { assess the rela- } \\
\text { tionship be- } \\
\text { tween Pap- } \\
\text { Smear testing } \\
\text { and independ- } \\
\text { ent variables }\end{array}$ & $\begin{array}{l}\text { 1. Non-refugee } \\
\text { women were sig- } \\
\text { nificantly more } \\
\text { likely to utilise } \\
\text { pap-screening } \\
\text { services than } \\
\text { refugee women2. } \\
\text { Significant predic- } \\
\text { tors of screening } \\
\text { uptake were work } \\
\text { arrangement, par- } \\
\text { ity, healthcare } \\
\text { visit, knowledge } \\
\text { and perceived } \\
\text { susceptibility of } \\
\text { cervical cancer }\end{array}$ \\
\hline $\begin{array}{l}\text { Anaman- } \\
\text { Torgbor, J } \\
\text { A. et al. }\end{array}$ & 2017 & $\begin{array}{l}\text { Detection } \\
\text { (Screening) }\end{array}$ & $\begin{array}{l}\text { Qualitative } \\
\text { Semi- } \\
\text { Structured } \\
\text { Interviews }\end{array}$ & $\begin{array}{l}\text { To describe } \\
\text { barriers and } \\
\text { facilitators of } \\
\text { cervical } \\
\text { screening } \\
\text { practices } \\
\text { among African } \\
\text { immigrant }\end{array}$ & $\begin{array}{l}\text { Brisbane, } \\
\text { Queensland, } \\
\text { Australia }\end{array}$ & $\begin{array}{l}19 \text { African } \\
\text { Immigrant } \\
\text { women; } 10 \\
\text { Refugee and } \\
9 \text { Non- } \\
\text { Refugee }\end{array}$ & $\begin{array}{l}19 \text { African } \\
\text { immigrant } \\
\text { women, aged } \\
\text { between } 21- \\
65 \text { years }\end{array}$ & $\begin{array}{l}\text { Interviews were } \\
\text { semi-structured } \\
\text { and transcribed } \\
\text { verbatim. They } \\
\text { were analysed } \\
\text { using interpret- } \\
\text { ive thematic } \\
\text { analysis. }\end{array}$ & $\begin{array}{l}\text { 1. Lack of } \\
\text { knowledge about } \\
\text { cervical cancer } \\
\text { and Pap smear, } \\
\text { the absence of } \\
\text { warning signs, } \\
\text { embarrassment, } \\
\text { fear, concern }\end{array}$ \\
\hline
\end{tabular}


Table. 2 Sample of data extraction and charting process (Continued)

\begin{tabular}{|c|c|c|c|c|c|c|c|c|c|}
\hline Author & Year & CCC Stage & $\begin{array}{l}\text { Study } \\
\text { Design }\end{array}$ & Aims/Purpose & Location & Sample Size & $\begin{array}{l}\text { Population } \\
\text { Group }\end{array}$ & Methodology & Key Findings \\
\hline & & & & $\begin{array}{l}\text { women living in } \\
\text { Brisbane, } \\
\text { Australia }\end{array}$ & & & & & $\begin{array}{l}\text { about the gender } \\
\text { of the service } \\
\text { provider, lack of } \\
\text { privacy, cultural } \\
\text { and religious } \\
\text { beliefs, and } \\
\text { healthcare system } \\
\text { factors were } \\
\text { identified as } \\
\text { barriers to } \\
\text { screening }\end{array}$ \\
\hline Author & Year & CCC Stage & $\begin{array}{l}\text { Study } \\
\text { Design }\end{array}$ & Aims/Purpose & Location & Sample Size & $\begin{array}{l}\text { Population } \\
\text { Group }\end{array}$ & Methodology & Key Findings \\
\hline $\begin{array}{l}\text { Cullerton, } \\
\text { K. et al. }\end{array}$ & 2016 & $\begin{array}{l}\text { Detection } \\
\text { (Screening) }\end{array}$ & $\begin{array}{l}\text { Cohort } \\
\text { study }\end{array}$ & $\begin{array}{l}\text { To understand } \\
\text { the impact of } \\
\text { education } \\
\text { sessions on the } \\
\text { knowledge and } \\
\text { attitudes } \\
\text { towards cancer } \\
\text { screening. }\end{array}$ & $\begin{array}{l}\text { Brisbane, } \\
\text { Queensland, } \\
\text { Australia }\end{array}$ & $\begin{array}{l}159 \\
\text { participants } \\
\text { in } 3 \\
\text { education } \\
\text { sessions }\end{array}$ & $\begin{array}{l}7 \text { CALD } \\
\text { groups; Arabic- } \\
\text { speaking, Bos- } \\
\text { nian, South } \\
\text { Asian, Samoan } \\
\text { and Pacific Is- } \\
\text { land, Spanish- } \\
\text { speaking, Su- } \\
\text { danese and } \\
\text { Vietnamese. }\end{array}$ & $\begin{array}{l}\text { All individuals } \\
\text { participated in } \\
\text { culturally } \\
\text { tailored cancer } \\
\text { screening } \\
\text { education } \\
\text { program and a } \\
\text { pre- and post- } \\
\text { education } \\
\text { evaluation mea- } \\
\text { sured changes } \\
\text { in knowledge, } \\
\text { attitudes and } \\
\text { intention related } \\
\text { to breast, bowel } \\
\text { and cervical } \\
\text { cancer and } \\
\text { screening }\end{array}$ & $\begin{array}{l}\text { 1. Overall } \\
\text { participants' } \\
\text { knowledge } \\
\text { increased, some } \\
\text { attitudes toward } \\
\text { participation in } \\
\text { cancer screening } \\
\text { became more } \\
\text { positive and } \\
\text { intent to } \\
\text { participate in } \\
\text { future screening } \\
\text { increased2. } \\
\text { Culturally tailored } \\
\text { education } \\
\text { programs are } \\
\text { effective in } \\
\text { improving } \\
\text { knowledge, } \\
\text { attitudes about } \\
\text { and intentions to } \\
\text { participate in } \\
\text { cancer screening }\end{array}$ \\
\hline $\begin{array}{l}\text { Kwok, } \\
\text { C.et al. }\end{array}$ & 2011 & $\begin{array}{l}\text { Detection } \\
\text { (Screening) }\end{array}$ & $\begin{array}{l}\text { Qualitative } \\
\text { Interviews }\end{array}$ & $\begin{array}{l}\text { To understand } \\
\text { the barriers and } \\
\text { facilitators to } \\
\text { cervical cancer } \\
\text { screening for } \\
\text { Chinese- } \\
\text { Australian } \\
\text { women }\end{array}$ & $\begin{array}{l}\text { New South } \\
\text { Wales, } \\
\text { Australia }\end{array}$ & $\begin{array}{l}18 \text { Chinese- } \\
\text { Australian } \\
\text { women }\end{array}$ & $\begin{array}{l}\text { Chinese- } \\
\text { Australian } \\
\text { women with } \\
\text { no history of } \\
\text { Cervical } \\
\text { Cancer }\end{array}$ & $\begin{array}{l}18 \text { women } \\
\text { participated in } \\
\text { qualitative } \\
\text { interviews in } \\
\text { their first } \\
\text { language } \\
\text { (Mandarin or } \\
\text { Cantonese) and } \\
\text { were analysed } \\
\text { using content } \\
\text { analysis }\end{array}$ & $\begin{array}{l}\text { 1. Knowledge of } \\
\text { Cervical Cancer } \\
\text { was low and few } \\
\text { participants } \\
\text { understood the } \\
\text { purpose of } \\
\text { screening2. } \\
\text { Having a doctor's } \\
\text { recommendation } \\
\text { was a strong } \\
\text { motivator, as was } \\
\text { having a female } \\
\text { Chinese doctor } \\
\text { and reminder } \\
\text { letter }\end{array}$ \\
\hline
\end{tabular}

incidence of clinical anxiety and depression [12, 62]. The third study examined Cantonese, Arabic and Mandarin speaking patients and found they experienced inequitable treatment quality due to the health services' expectation of English proficiency and familiarity with the health system. This resulted in diminished understandings and explanations about cancer, treatment and the roles of specialists [5]. Two additional studies found
CALD populations were significantly underrepresented, or not clearly represented in clinical trial research and participation $[61,64]$.

\section{Experiences of survivorship}

Seven studies discussed the stage of survivorship for CALD populations [68-74]. Six were qualitative interviews or focus groups $[68,69,71-74]$ and one cohort 

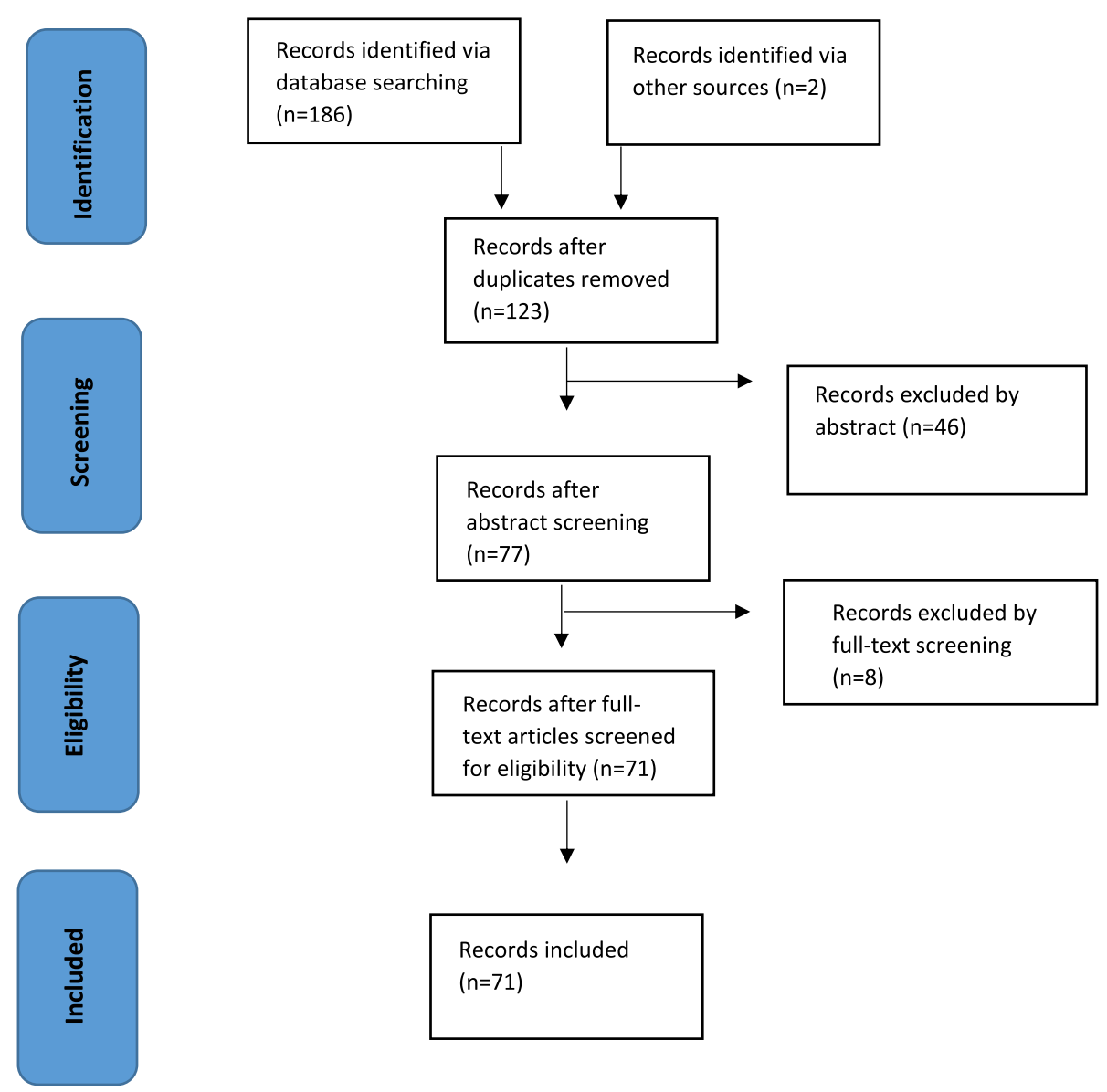

Records included

$(n=71)$

Fig. 2 Search process illustrated in a PRISMA flowchart for scoping review

study [70]. Four of the qualitative studies retrospectively described experiences of inequity during cancer treatment. This displayed issues with incorrect interpreter usage, a greater need for information to manage illness and further explanations about tests and side effects prior to undergoing treatment $[68,69]$. Similarly, Butow et al. (2013) found 'immigrant cancer survivors' were more likely to report unmet physical care or informational needs [70]. Ongoing cancer-related stressors in the survivorship stage, such as lack of culturally or linguistically specific survivorship information and resources, difficulty navigating health system and community entitlements and lack of appropriate caregiver information, were also reported [70, 72]. Studies further reported a reliance on family members and bilingual general practitioners for effective survivorship care coordination and a desire for acknowledgement of the diversity of survivorship experiences between CALD groups $[72,73]$.

\section{Palliative care}

There were limited studies related to equity during palliative care for CALD populations and their families, with only three studies reporting on this [75-77]. In qualitative interviews with CALD patients and their families during the palliative care phase, Kirby et al. (2018) found issues during the transition to palliative care, such as poor communication about patient management and individuals not wishing to discuss death and dying directly, and highlighted the importance of cultural and spiritual needs [77]. A retrospective study examined a cohort of deceased CALD patients to examine the physical and psychological journey at end-of-life [76]. This found non-English speaking patients did not receive equitable assessment of physical symptoms at end-of-life [76]. Additionally, those who identified the need for an interpreter on admission, only $9 \%$ accessed professional interpreters throughout their admission [76]. Additionally, poor documentation of cultural considerations was common, with post-death care of the patient's body documented in only $20 \%$ of cases [76].

\section{Communication}

Poor communication across the cancer care continuum was a consistent theme, with eight studies discussing it 
directly [13, 78-84]. Communication was described as a significant barrier to equitable care, with migrants expressing feeling alone and misunderstood by health services $[13,80]$. A lack of consistency with interpreters led to many feeling unable to understand medical instructions or communicate issues and concerns with health providers $[13,80]$. A cohort study compared the oncology consultations of 'immigrant' patients, with and without interpreters, to that of Australian-born patients [78]. Findings showed doctors spoke less to immigrant patients with interpreters than to 'Anglo-Australians', spent proportionally less time discussing, summarising and informing on cancer-related issues, and tended to delay or omit more responses to immigrant patients [78]. This was thought to be due to the time constraints of repeating questions and responses through interpreters, as well as the incorrect assumption by some clinicians that ethnically diverse individuals prefer a paternalistic approach to communication. This was shown to be incorrect during post-consultation interviews [78].

\section{Patient and clinician perspectives}

Three studies investigated the perspectives of clinicians directly [85-87] and four examined the perspectives of patients [88-91]. Clinicians reported limited culturallyappropriate translated resources, difficulty engaging appropriate interpreters, lack of funding, a culture of "learning on the job" and time constraints as significant structural barriers to providing equitable care for CALD populations $[86,87]$. The importance of prioritising and developing quality relationships with CALD patients was highlighted in clinician focus groups as a facilitator of effective intercultural care [85]. Medical clinicians reported the tendency to refer complex culturally diverse patients to allied health or multicultural health workers, thus limiting their access to specialist oncology clinicians [85]. Patients reported wanting more information about cancer, as well as diagnostic and treatment options [89]. Lacking information was a persistent theme, with two further studies highlighting migrant women are significantly less likely to undergo breast reconstruction postmastectomy, often due to a lack of information and counselling [88, 91]. Patients reported challenges communicating with health professionals and that many resources did not cater to those with limited English skills $[89,90]$.

\section{Discussion}

This study presents a comprehensive review of the published literature regarding equity across the cancer care continuum for CALD migrant populations living in Australia. A key finding is the persistent focus within the literature on patient-sided barriers to care. This places a disproportionate level of burden on those who experience health inequities and obscures the structural, social and political processes that produce health inequities [92]. The lack of critique evident in the included studies shows researchers are also contributing to an individualistic, deficit perspective of health equity.

A key finding of this review is the role of health systems in creating and reinforcing cancer inequities. This is exemplified through pervasive communication problems across the cancer care continuum, a lack of culturally and linguistically appropriate cancer and treatment related information and a health system that is difficult for patients to navigate. In addition, clinicians report a context of inadequate support, resources and significant time constraints that restrict their ability to provide equitable care. These factors create a situation where CALD migrant populations are less informed about their health and treatment options, have difficulty communicating their concerns, find health services challenging to navigate and receive poorer quality care across many areas of the cancer care continuum [86, 87, 89, 93]. This demonstrates a culturally dominant model of care is not adequate in promoting equitable care for all populations and that targeted, culturally and linguistically responsive services, which support both patients and clinicians, are critical to equity [4].

Despite being a multilingual and multi-ethnic society, Australian health institutions have yet to take the necessary steps to move beyond the culturally dominant model of care. This model causes marginalisation of ethnic and cultural minority populations and reinforces assumptions and practices that lead to health inequities. This review also highlighted the need to provide further resources and training for clinicians, particularly in the development of the meaningful relationships needed to provide effective intercultural care [85]. It has been reported through focus-groups with clinicians that a lack of high-level interpreter services impedes their ability to assess patient symptoms and to develop intimate and trusting relationships with CALD patients [94]. This review also highlighted the need to expand health equity research into the field of palliative care. The limited Australian literature indicates significant challenges to achieving equitable and culturally appropriate palliative care [95]. Communication problems and a lack of attention to diverse values and practices at end-of-life have been highlighted as significant concerns [95]. Therefore, there is a need to investigate and establish new modes of caring that engage patients, families and communities [77].

This study revealed an uneven distribution of research spanning the cancer care continuum and an overreliance on screening attendance rates as a measure of equity. This is significant because there are many 
cancers for which screening is not recommended or considered beneficial to patient outcomes [96]. Therefore, it can be argued that focus on other phases of the cancer care continuum, such as diagnostic pathways and treatment outcomes may have a greater influence on health equity for CALD migrant populations.

Few studies directly compared equity in access or outcomes, with most qualitative studies focusing on healthcare experiences or preferences of individuals. This is significant as there is growing acknowledgement that behavioral explanations of health inequities are inadequate and there is a need to examine the structural and institutional drivers of inequity [92]. For example, studies that describe patient-sided barriers to care, such as lack of English proficiency, are often describing institutional inaccessibility [5]. The expectation that patients speak English and can navigate the health system directly reflects the privileged positioning of majority groups and the marginalised positioning of others [97].

This review focused on equity within the Australian context, but highlights issues of global concern. With aging populations and an increased cancer burden in both OECD and emerging economies [7-9], the need for standardised equity indictors across the cancer care continuum is clear. This would allow for an expansion of research across the cancer care continuum and allow international health services to respond appropriately to the demographic and epidemiological changes associated with current and future migration flows [3, 7].

Due to the relatively small number of studies in each stage of the cancer care continuum and the heterogeneity of those studies, no conclusive findings can be taken from this review.

An important limitation to consider is that only studies published in English were included and as such many relevant studies in languages other than English may have been excluded. Additionally, most studies restricted populations to a small number of CALD groups, such as Chinese, Arabic and Greek speaking populations and therefore findings may not be generalisable across migrant groups.

\section{Conclusions}

This study demonstrates that few studies have comprehensively assessed equity across the cancer continuum for CALD migrant populations in Australia. Within the current literature, there is a significant lack of critique examining the social, structural and institutional drivers of inequity. A further examination of power differentials, social positioning, marginalisation and the impact of majoritarian histories on health services will provide a deeper insight into the operationalisation of health equity. Research to date has placed a disproportionate burden on those who experience health inequities, rather than examining entrenched power differentials and institutional processes. It is necessary that health services shift their focus to the promotion of equity in order to become responsive to the diverse and changing needs of their populations.

\section{Abbreviations \\ CALD: Culturally and linguistically diverse; CHB: Chronic Hepatitis B; $\mathrm{H}$. pylori: Helicobacter pylori}

\section{Acknowledgements \\ The authors would like to gratefully acknowledge Naomi Stekelenburg, the Queensland University of Technology Researcher Advocate, for her assistance in the pre-submission editing of this manuscript. Her expertise is greatly} appreciated.

\section{Authors' contributions}

BS conducted the scoping review under the guidance of JD and MB. DW gave specialist clinical guidance. Data were analysed by BS and BS was the major contributor for the writing of the manuscript. All authors read and approved the final manuscript.

\section{Funding}

This review was possible through the lead authors Royal Brisbane and Women's Hospital post-graduate scholarship.

Availability for data and materials

The datasets used and/or analysed during the current study are available from the corresponding author on reasonable request: b.scanlon@uq.net.au.

\section{Declarations}

Ethics approval and consent to participate Not applicable.

\section{Consent for publication}

Not applicable.

\section{Competing interest}

The authors declare that they have no competing interests.

\section{Author details}

${ }^{1}$ Queensland University of Technology, 149 Victoria Park Road, QLD 4059 Kelvin Grove, Australia. ${ }^{2}$ Royal Brisbane and Women's Hospital, Butterfield Street, QLD 4029 Herston, Australia. ${ }^{3}$ University of Queensland, 20 Weightman Street, QLD 4006 Herston, Australia.

Received: 24 March 2021 Accepted: 8 July 2021

Published online: 28 July 2021

\section{References}

1. Chiriboga D, Garay J, Buss P, Madrigal RS, Rispel LC. Health inequity during the COVID-19 pandemic: a cry for ethical global leadership. Lancet. 2020; 395(10238):1690-1.

2. Luiking ML, Heckemann B, Ali P, Dekker-van Doorn C, Ghosh S, Kydd A, et al. Migrants' Healthcare Experience: A Meta-Ethnography Review of the Literature. J Nurs Scholarsh. 2019;51(1):58-67.

3. MIGRATION AND MIGRANTS: A GLOBAL OVERVIEW: International Organization for Migration; 2020 [Available from: https://publications.iom. int/system/files/pdf/wmr_2020_en_ch_2.pdf.

4. Griffith DM, Shelton RC, Kegler M. Advancing the Science of Qualitative Research to Promote Health Equity. Health Educ Behav. 2017;44(5):673-6.

5. Shaw J, Butow P, Sze M, Young J, Goldstein D. Reducing disparity in outcomes for immigrants with cancer: a qualitative assessment of the feasibility and acceptability of a culturally targeted telephone-based supportive care intervention. Support Care Cancer. 2013;21(8):2297-301.

6. Vekic B, Dragojevic-Simic V, Jakovljevic M, Kalezic M, Zagorac Z, Dragovic S, et al. A Correlation Study of the Colorectal Cancer Statistics and Economic Indicators in Selected Balkan Countries. Front Public Health. 2020;8:29. 
7. Global Burden of Disease. Cancer C, Fitzmaurice C, Akinyemiju TF, Al Lami FH, Alam T, Alizadeh-Navaei R, et al. Global, Regional, and National Cancer Incidence, Mortality, Years of Life Lost, Years Lived With Disability, and Disability-Adjusted Life-Years for 29 Cancer Groups, 1990 to 2016: A Systematic Analysis for the Global Burden of Disease Study. JAMA Oncol. 2018;4(11):1553-68.

8. Jakovljevic MB, Milovanovic O. Growing Burden of Non-Communicable Diseases in the Emerging Health Markets: The Case of BRICS. Front Public Health. 2015;3:65.

9. Lamnisos D, Giannakou K, Jakovljevic MM. Demographic forecasting of population aging in Greece and Cyprus: one big challenge for the Mediterranean health and social system long-term sustainability. Health Res Policy Syst. 2021;19(1):21.

10. Global Burden of Disease. Cancer C, Fitzmaurice C, Allen C, Barber RM, Barregard L, Bhutta ZA, et al. Global, Regional, and National Cancer Incidence, Mortality, Years of Life Lost, Years Lived With Disability, and Disability-Adjusted Life-years for 32 Cancer Groups, 1990 to 2015: A Systematic Analysis for the Global Burden of Disease Study. JAMA Oncol. 2017:3(4):524-48.

11. Brain GBD, Other CNSCC. Global, regional, and national burden of brain and other CNS cancer, 1990-2016: a systematic analysis for the Global Burden of Disease Study 2016. Lancet Neurol. 2019;18(4):376-93.

12. Sze M, Butow $P$, Bell M, Vaccaro L, Dong S, Eisenbruch M, et al. Migrant health in cancer: outcome disparities and the determinant role of migrantspecific variables. Oncologist. 2015;20(5):523-31.

13. Hyatt A, Lipson-Smith R, Schofield P, Gough $K$, Sze M, Aldridge L, et al. Communication challenges experienced by migrants with cancer: A comparison of migrant and English-speaking Australian-born cancer patients. Health Expect. 2017;20(5):886-95

14. Wang K, Hendrickson Z, Brandt C, Nunez-Smith M. The relationship between non-permanent migration and non-communicable chronic disease outcomes for cancer, heart disease and diabetes - a systematic review. BMC Public Health. 2019;19(1):1-13.

15. Cancer Health Disparities in the United States- Facts and Figures [Internet]. American Society of Clinical Oncology. 2015. [cited 26 April 2020]. Available from: https://www.cancer.net/sites/cancer.net/files/health_disparities_fact_ sheet.pdf.

16. Thomas BC, Carlson LE, Bultz BD. Cancer patient ethnicity and associations with emotional distress-the 6th vital sign: a new look at defining patient ethnicity in a multicultural context. J Immigr Minor Health. 2009;11(4):237-48.

17. Wang AMQ, Yung EM, Nitti N, Shakya Y, Alamgir AKM, Lofters AK. Breast and Colorectal Cancer Screening Barriers Among Immigrants and Refugees: A Mixed-Methods Study at Three Community Health Centres in Toronto, Canada. J Immigr Minor Health. 2019;21(3):473-82.

18. Disparities | Healthy People 2020 [Internet]. Healthypeople.gov. 2014 [cited 24 April 2020]. Available from: https://www.healthypeople.gov/2020/about/ foundation-health-measures/Disparities.

19. Veenstra G. Racialized identity and health in Canada: results from a nationally representative survey. Soc Sci Med. 2009;69(4):538-42.

20. Butow $P$, Sze $M$, Dugal-Beri $P$, Mikhail $M$, Eisenbruch $M$, Jefford $M$, et al. From inside the bubble: migrants' perceptions of communication with the cancer team. Support Care Cancer. 2011;19(2):281-90.

21. Shinagawa SM. The excess burden of breast carcinoma in minority and medically underserved communities: application, research, and redressing institutional racism. Cancer. 2000;88(5 Suppl):1217-23.

22. Braveman P. What are health disparities and health equity? We need to be clear. Public Health Rep. 2014;129(Suppl 2):5-8

23. Braveman $P$. What is health equity: and how does a life-course approach take us further toward it? Matern Child Health J. 2014;18(2):366-72.

24. Cancer Control Continuum | Division of Cancer Control and Population Sciences (DCCPS) [Internet]. Cancercontrol.cancer.gov. 2020. [cited 26 April 2020]. Available from: https://cancercontrol.cancer.gov/about-dccps/about$\mathrm{cc} /$ cancer-control-continuum.

25. Chapter 11: Scoping reviews - JBI Manual for Evidence Synthesis - JBI GLOBAL WIKI [Internet]. Wiki.jbi.global. 2020. [cited 24 April 2020]. Available from: https://wiki.jbi.global/display/MANUAL/Chapter+11\%3A+Scoping+ reviews.

26. Zhou Q. Accessing disability services by people from culturally and linguistically diverse backgrounds in Australia. Disabil Rehabil. 2016:38(9): 844-52.
27. Robotin MC, Kansil MQ, George J, Howard K, Tipper S, Levy M, et al. Using a population-based approach to prevent hepatocellular cancer in New South Wales, Australia: effects on health services utilisation. BMC Health Serv Res. 2010;10:215-.

28. Schulz TR, McBryde ES, Leder K, Biggs B-A. Using stool antigen to screen for Helicobacter pylori in immigrants and refugees from high prevalence countries is relatively cost effective in reducing the burden of gastric cancer and peptic ulceration. PLoS One. 2014;9(9):e108610-e.

29. Parajuli J, Horey D, Avgoulas M. Best practice models recommended by Bhutanese refugee women for cervical and breast cancer screening in Australia: A qualitative study. Health Promot J Austr. 2020;31(3):381-90.

30. Anaman-Torgbor JA, King J, Correa-Velez I. Barriers and facilitators of cervical cancer screening practices among African immigrant women living in Brisbane, Australia. Eur J Oncol Nurs. 2017;31:22-9.

31. Javanparast S, Ward PR, Carter SM, Wilson CJ. Barriers to and facilitators of colorectal cancer screening in different population subgroups in Adelaide, South Australia. Med J Aust. 2012;196(8):521-3.

32. O'Hara J, McPhee C, Dodson S, Cooper A, Wildey C, Hawkins M, et al. Barriers to Breast Cancer Screening among Diverse Cultural Groups in Melbourne, Australia. Int J Environ Res Public Health. 2018;15(8):1677.

33. Ogunsiji OO, Kwok C, Fan LC. Breast cancer screening practices of African migrant women in Australia: a descriptive cross-sectional study. BMC Womens Health. 2017;17(1):32-.

34. Weber MF, Banks E, Smith DP, O'Connell D, Sitas F. Cancer screening among migrants in an Australian cohort; cross-sectional analyses from the 45 and Up Study. Bmc Public Health. 2009;9:144-

35. Cullerton K, Gallegos D, Ashley E, Do H, Voloschenko A, Fleming M, et al. Cancer screening education: can it change knowledge and attitudes among culturally and linguistically diverse communities in Queensland. Australia? Health Promot J Austr. 2016;27(2):140-7.

36. Taylor RJ, Morrell SL, Mamoon HA, Macansh S, Ross J, Wain GV. Cervical cancer screening in a Vietnamese nominal cohort. Ethn Health. 2003:8(3): 251-61

37. Aminisani N, Armstrong BK, Canfell K. Cervical cancer screening in Middle Eastern and Asian migrants to Australia: a record linkage study. Cancer Epidemiol. 2012;36(6):e394-400.

38. Taylor RJ, Mamoon HA, Morrell SL, Wain GV. Cervical screening in migrants to Australia. Aust N Z J Public Health. 2001;25(1):55-61.

39. Kwok C, White K, Roydhouse JK. Chinese-Australian women's knowledge, facilitators and barriers related to cervical cancer screening: a qualitative study. Journal of immigrant minority health. 2011;13(6):1076-83.

40. Suwankhong D, Liamputtong P. Early Detection of Breast Cancer and Barrier to Screening Programmes amongst Thai Migrant Women in Australia: A Qualitative Study. Asian Pac J Cancer Prev. 2018;19(4):1089-97.

41. Phillipson L, Pitts L, Hall J, Tubaro T. Factors contributing to low readiness and capacity of culturally diverse participants to use the Australian national bowel screening kit. Public Health Res Pract. 2019;29(1):28231810.

42. Jirojwong S, Maclennan R, Manderson L. Health beliefs and Pap smears among Thai women in Brisbane, Australia. Asia Pac J Public Health. 2001; 13(1):20-3.

43. Jirojwong S, MacLennan R. Health beliefs, perceived self-efficacy, and breast self-examination among Thai migrants in Brisbane. J Adv Nurs. 2003;41(3): 241-9.

44. Aminisani N, Armstrong BK, Egger S, Canfell K. Impact of organised cervical screening on cervical cancer incidence and mortality in migrant women in Australia. BMC Cancer. 2012;12:491-.

45. Anaman JA, Correa-Velez I, King J. Knowledge Adequacy on Cervical Cancer Among African Refugee and Non-Refugee Women in Brisbane, Australia. J Cancer Educ. 2018;33(3):716-23.

46. Ogunsiji O, Wilkes L, Peters K, Jackson D. Knowledge, attitudes and usage of cancer screening among West African migrant women. J Clin Nurs. 2013; 22(7-8):1026-33.

47. Aminisani N, Armstrong BK, Canfell K. Participation in cervical screening by older asian and middle eastern migrants in new South wales, australia. Health Promot Perspect. 2012;2(2):274-86.

48. Lam M, Kwok C, Lee M-J. Prevalence and sociodemographic correlates of routine breast cancer screening practices among migrant-Australian women. Aust N Z J Public Health. 2018:42(1):98-103.

49. Anaman JA, Correa-Velez I, King J. A survey of cervical screening among refugee and non-refugee African immigrant women in Brisbane, Australia. Health Promot J Austr. 2017;28(3):217-24. 
50. Parajuli J, Horey D, Avgoulas M-I. Access to breast cancer screening -perception, and perceived barriers among older Bhutanese refugee women resettled in Australia: a qualitative study. Australian Journal of Cancer Nursing. 2019;20(1):14-8.

51. Mazza D, Lin X, Walter FM, Young JM, Barnes DJ, Mitchell P, et al. The LEAD study protocol: a mixed-method cohort study evaluating the lung cancer diagnostic and pre-treatment pathways of patients from Culturally and Linguistically Diverse (CALD) backgrounds compared to patients from Anglo-Australian backgrounds. BMC Cancer. 2018;18(1):754-

52. Beauchamp A, Mohebbi M, Cooper A, Pridmore V, Livingston P, Scanlon M, et al. The impact of translated reminder letters and phone calls on mammography screening booking rates: Two randomised controlled trials. PLoS One. 2020;15(1):e0226610-e.

53. Bell ML, Butow PN, Goldstein D. Informatively missing quality of life and unmet needs sex data for immigrant and Anglo-Australian cancer patients and survivors. Qual Life Res. 2013;22(10):2757-60.

54. Chiang Y-C, Collins A, Chopra P, Lu T, Tan E-S, Couper JW. Understanding the experiences of Mandarin-speaking patients diagnosed with lifethreatening cancer in Australia. Palliat Support Care. 2015;13(5):1317-23.

55. Goldstein D, Bell ML, Butow P, Sze M, Vaccaro L, Dong S, et al. Immigrants' perceptions of the quality of their cancer care: an Australian comparative study, identifying potentially modifiable factors. Ann Oncol. 2014;25(8):1643-9.

56. Hwang E, Yap ML, Boxer MM, Chong S, Duggan K, Kaadan N, et al. Variations in breast cancer histology and treatment patterns between the major ethnic groups of South West Sydney. Breast J. 2018;24(4):615-9.

57. Hyatt A, Lipson-Smith R, Gough K, Butow P, Jefford M, Hack TF, et al. Culturally and linguistically diverse oncology patients' perspectives of consultation audio-recordings and question prompt lists. Psychooncology. 2018;27(9):2180-8

58. Lim BT, Butow P, Mills J, Miller A, Pearce A, Goldstein D. Challenges and perceived unmet needs of Chinese migrants affected by cancer: Focus group findings. J Psychosoc Oncol. 2019;37(3):383-97.

59. Lim B, Butow P, Sze M, Girgis A, Jefford M, Goldstein D, et al. Impact of migrancy on cancer clinical trial participation: Factors associated with approach and consent in Australian-born versus migrant groups. Asia Pac J Clin Oncol. 2020;16(3):115-22.

60. Lim BT, Huang YJ, Shepherd HL, Shaw J, Costa D, Durcinoska I, et al. Health literacy and cancer care coordination in Chinese migrant patients and their carers: A cross-sectional survey. Psychooncology. 2019;28(5):1048-55.

61. Lwin Z, Broom A, Cosman R, Livingstone A, Sawkins K, Good P, et al. Culturally and linguistically diverse patient participation in glioma research. Neurooncol Pract. 2014;1(3):101-5.

62. Ming S, Butow P, Bell M, Vaccaro L, Dong S, Eisenbruch M, et al. Migrant Health in Cancer: Outcome Disparities and the Determinant Role of Migrant-Specific Variables. Oncologist. 2015;20(5):523-31.

63. Shaw JM, Shepherd HL, Durcinoska I, Butow PN, Liauw W, Goldstein D, et al. It's all good on the surface: care coordination experiences of migrant cancer patients in Australia. Support Care Cancer. 2016;24(6):2403-10.

64. Smith AB, Agar M, Delaney G, Descallar J, Dobell-Brown K, Grand M, et al. Lower trial participation by culturally and linguistically diverse (CALD) cancer patients is largely due to language barriers. Asia Pac J Clin Oncol. 2018;14(1):52-60.

65. Thai AA, Tacey M, Byrne A, White S, Yoong J. Exploring disparities in receipt of adjuvant chemotherapy in culturally and linguistically diverse groups: an Australian centre's experience. Intern Med J. 2018;48(5):561-6.

66. Shaw J, Zou X, Butow P. Treatment decision making experiences of migrant cancer patients and their families in Australia. Patient Educ Couns. 2015; 98(6):742-7.

67. Young JM, Venchiarutti RL, Durcinoska I, Steffens D. Cross-Cultural Adaptation And Pilot Testing Of The Cancer Care Coordination Questionnaire For Patients (CCCQ-P) In Chinese And Arabic Languages. Patient Prefer Adherence. 2019;13:1791-7.

68. Alananzeh I, Ramjan L, Kwok C, Levesque JV, Everett B. Arab-migrant Cancer Survivors' Experiences of Using Health-care Interpreters: A Qualitative Study. Asia Pac J Oncol Nurs. 2018;5(4):399-407.

69. Alananzeh IM, Kwok C, Ramjan L, Levesque JV, Everett B. Information needs of Arab cancer survivors and caregivers: A mixed methods study. Collegian. 2019;26(1):40-8.

70. Butow PN, Bell ML, Aldridge LJ, Sze M, Eisenbruch M, Jefford M, et al. Unmet needs in immigrant cancer survivors: a cross-sectional populationbased study. Support Care Cancer. 2013;21(9):2509-20.
71. Hunter J, Ussher J, Parton C, Kellett A, Smith C, Delaney G, et al. Australian integrative oncology services: a mixed-method study exploring the views of cancer survivors. BMC Complement Altern Med. 2018;18(1):153-.

72. O'Callaghan C, Schofield P, Butow P, Nolte L, Price M, Tsintziras S, et al. "I might not have cancer if you didn't mention it": a qualitative study on information needed by culturally diverse cancer survivors. Support Care Cancer. 2015;24(1):409-18.

73. Tan L, Gallego G, Nguyen TTC, Bokey L, Reath J. Perceptions of shared care among survivors of colorectal cancer from non-English-speaking and English-speaking backgrounds: a qualitative study. BMC Fam Pract. 2018; 19(1):134-.

74. Wiley G, Piper A, Phyllis Butow AM, Schofield P, Douglas F, Roy J, et al. Developing Written Information for Cancer Survivors from Culturally and Linguistically Diverse Backgrounds: Lessons Learnt. Asia Pac J Oncol Nurs. 2018;5(1):121-6.

75. Green A, Jerzmanowska N, Green M, Lobb E. 'Death is difficult in any language': A qualitative study of palliative care professionals' experiences when providing end-of-life care to patients from culturally and linguistically diverse backgrounds. Palliat Med. 2018;32(8):1419-27.

76. Green A, Jerzmanowska N, Thristiawati S, Green M, Lobb E. Culturally and linguistically diverse palliative care patients' journeys at the end-of-life. Palliat Support Care. 2018;17(2):227-33.

77. Kirby E, Lwin Z, Kenny K, Broom A, Birman H, Good P. "It doesn't exist...": negotiating palliative care from a culturally and linguistically diverse patient and caregiver perspective. BMC Palliat Care. 2018;17(1):1-10.

78. Butow P, Bell M, Goldstein D, Sze M, Aldridge L, Abdo S, et al. Grappling with cultural differences; communication between oncologists and immigrant cancer patients with and without interpreters. Patient Educ Couns. 2011;84(3):398-405

79. Butow PN, Lobb E, Jefford M, Goldstein D, Eisenbruch M, Girgis A, et al. A bridge between cultures: interpreters' perspectives of consultations with migrant oncology patients. Support Care Cancer. 2012;20(2):235-44.

80. Butow PN, Sze M, Dugal-Beri P, Mikhail M, Eisenbruch M, Jefford M, et al. From inside the bubble: migrants' perceptions of communication with the cancer team. Support Care Cancer. 2010;19(2):281-90.

81. Lipson-Smith R, Hyatt A, Butow P, Hack TF, Jefford M, Hale S, et al. Are audio recordings the answer? - a pilot study of a communication intervention for non-English speaking patients with cancer. Psychooncology. 2016;25(10):1237-40.

82. Lipson-Smith R, Hyatt A, Murray A, Butow P, Hack TF, Jefford M, et al. Measuring recall of medical information in non-English-speaking people with cancer: A methodology. Health Expect. 2018;21(1):288-99.

83. McGrane JA, Butow PN, Sze M, Eisenbruch M, Goldstein D, King MT. Assessing the invariance of a culturally competent multi-lingual unmet needs survey for immigrant and Australian-born cancer patients: a Rasch analysis. Qual Life Res. 2014;23(10):2819-30.

84. Mitchison D, Butow P, Sze M, Aldridge L, Hui R, Vardy J, et al. Prognostic communication preferences of migrant patients and their relatives. Psychooncology. 2012;21(5):496-504.

85. Broom A, Parker RB, Kirby E, Kokanović R, Woodland L, Lwin Z, et al. A qualitative study of cancer care professionals' experiences of working with migrant patients from diverse cultural backgrounds. BMJ Open. 2019;9(3): e025956-e.

86. Saleh M, Barlow-Stewart K, Meiser B, Muchamore I. Challenges faced by genetics service providers' practicing in a culturally and linguistically diverse population: An Australian experience. Journal of Genetic Counseling. 2009; 18(5):436-46

87. Watts K, Meiser B, Zilliacus E, Kaur R, Taouk M, Girgis A, et al. Perspectives of oncology nurses and oncologists regarding barriers to working with patients from a minority background: Systemic issues and working with interpreters. Eur J Cancer Care (Engl). 2018;27(2):e12758-e.

88. Mo HTJ, Segara D, Yarrow S, Soon PS, Girgis A, Ruban S, et al. Understanding patient choices regarding breast reconstruction after mastectomy for breast cancer. Support Care Cancer. 2019;27(6):2135-42.

89. Robotin MC, Porwal M, Hopwood M, Nguyen D, Sze M, Treloar C, et al. Listening to the consumer voice: developing multilingual cancer information resources for people affected by liver cancer. Health Expect. 2017;20(1):171-82

90. Scott N, Donato-Hunt C, Crane M, Lafontaine M, Varlow M, Seale H, et al. Knowledge, attitudes and beliefs about lung cancer in three culturally and 
linguistically diverse communities living in Australia: a qualitative study. Health Promot J Austr. 2014;25(1):46-51.

91. Soon P, Karimi N, Wu V, Girgis A. Having breast reconstruction postmastectomy: barriers and facilitators reported by Vietnamese- versus English-speaking women with breast cancer. Ethn Health. 2019;2(1):1-18.

92. Viruell-Fuentes EA, Miranda PY, Abdulrahim S. More than culture: structural racism, intersectionality theory, and immigrant health. Soc Sci Med. 2012; 75(12):2099-106.

93. Broom A, Parker R, Raymond S, Kirby E, Lewis S, Wyld D, et al. The (Co)Production of Difference in the Care of Patients With Cancer From Migrant Backgrounds. Qual Health Res. 2020;30(11):1619-31.

94. Broom A, Kirby E, Kokanovic R, Woodland L, Wyld D, de Souza P, et al. Individualising difference, negotiating culture: Intersections of culture and care. Health. 2020;24(5):552-71.

95. Broom A, Good P, Kirby E, Lwin Z. Negotiating palliative care in the context of culturally and linguistically diverse patients. Intern Med J. 2013;43(9): 1043-6.

96. Hoffmann TC, Del Mar C. Patients' expectations of the benefits and harms of treatments, screening, and tests: a systematic review. JAMA Intern Med. 2015;175(2):274-86.

97. Ford $\mathrm{CL}$, Airhihenbuwa CO. The public health critical race methodology: praxis for antiracism research. Soc Sci Med. 2010;71(8):1390-8.

\section{Publisher's Note}

Springer Nature remains neutral with regard to jurisdictional claims in published maps and institutional affiliations.

Ready to submit your research? Choose BMC and benefit from:

- fast, convenient online submission

- thorough peer review by experienced researchers in your field

- rapid publication on acceptance

- support for research data, including large and complex data types

- gold Open Access which fosters wider collaboration and increased citations

- maximum visibility for your research: over $100 \mathrm{M}$ website views per year

At $\mathrm{BMC}$, research is always in progress.

Learn more biomedcentral.com/submissions 